\title{
Recent VBS scattering measurements with two vector bosons in the final state
}

\author{
Flavia Cetorelli* on behalf of the CMS Collaboration \\ Dipartimento di Fisica "Giuseppe Occhialini", Università degli Studi di Milano - Bicocca, \\ Piazza della Scienza 3, Milano, Italia \\ INFN Sezione di Milano - Bicocca, \\ Piazza della Scienza 3, Milano, Italia \\ E-mail: f.cetorelli@campus.unimib.it
}

\begin{abstract}
We summarize the latest cross-section measurements of final states with two vector bosons and two jets, produced in Vector Boson Scattering (VBS) processes. The analyses are based on the LHC proton-proton collisions at a center-of-mass energy of $13 \mathrm{TeV}$ recorded with the CMS detector. VBS processes are powerful tools to shed light on the mechanism underlying the spontaneous breaking of the electroweak symmetry. Indeed, the unitarization of their cross sections is strictly related to the Higgs boson; any possible deviation from standard model predictions could lead to an enhancement of their cross sections, which could reveal the nature of what lies beyond the standard model.
\end{abstract}

\footnotetext{
*** The European Physical Society Conference on High Energy Physics (EPS-HEP2021), ***

*** 26-30 July 2021 ***

*** Online conference, jointly organized by Universität Hamburg and the research center DESY ***
}

\footnotetext{
${ }^{*}$ Speaker
} 


\section{Introduction}

Vector boson scattering (VBS) processes are among the rarest productions measured at the LHC, as they are mediated by purely electroweak (EW) interaction at the tree level. Due to their strong connection to the Higgs boson, which guarantees the unitarity of their cross sections, VBS processes allow one to investigate the EW symmetry-breaking mechanism at high energy scales. Polarization studies involving the longitudinally polarized gauge bosons are particularly suitable for this task. Moreover, VBS processes are fundamental tools in the detection of new physics. Indeed, they are proper candidates for constraining the anomalous triple (aTGCs) and quartic gauge couplings (aQGCs) described in the effective field theory (EFT) approach.

The VBS final state is characterized by the presence of two jets (jj), which originate from initial state partons. These VBS jets are well separated in pseudorapidity $\left(\Delta \eta_{j j}\right)$ and have a high dijet invariant mass $\left(m_{j j}\right)$. The two vector bosons produced, if massive, may decay in a pair of leptons (leptonic decay) or a pair of quarks (hadronic decay). The purely leptonic final state, in which all the massive bosons decayed leptonically, although having a smaller branching fraction than the other ones, benefits an enhanced signal purity due to less background contamination.

At the leading order (LO), the production of two vector bosons in association with two jets may also arise from processes of order $\alpha_{S}^{2} \alpha^{2}$, where the interaction between the two initial state quarks is mediated by the quantum chromodynamics (QCD). This process constitutes an irreducible background, as it produces the same final state as the EW signal.

\section{VBS in CMS with the $13 \mathrm{TeV}$ Full Run II data set}

\subsection{The golden channel: First polarization measurements}

The EW production of two $\mathrm{W}$ bosons with the same sign charge in association with two jets $\left(\mathrm{W}^{ \pm} \mathrm{W}^{ \pm} \mathrm{jj}\right)$ is known as "the golden channel" among the VBS processes; indeed, it has the lowest contamination from QCD-induced background. In a recent publication from CMS [1], the fully leptonic final state is chosen to investigate the polarized cross sections of this process [2].

The analysis selects events requiring exactly two leptons (electrons or muons) with a charge of the same sign, moderate missing transverse momentum, and two jets with the peculiar VBS characteristics. Two different binned maximum-likelihood (ML) fits are performed: one to measure $\mathrm{W}_{\mathrm{L}}^{ \pm} \mathrm{W}_{\mathrm{L}}^{ \pm} / \mathrm{W}_{\mathrm{X}}^{ \pm} \mathrm{W}_{\mathrm{T}}^{ \pm}$components, the other to measure the $\mathrm{W}_{\mathrm{L}}^{ \pm} \mathrm{W}_{\mathrm{X}}^{ \pm} / \mathrm{W}_{\mathrm{T}}^{ \pm} \mathrm{W}_{\mathrm{T}}^{ \pm}$processes. Here, the index $\mathrm{L}$ indicates the longitudinal polarization state, the $\mathrm{T}$ the transverse one, while the $\mathrm{X}$ can represent either an L or a T polarization state. Two signal boosted decision trees (BDT) (Fig. 1) separate the various polarized components from each other, whereas an inclusive BDT disentangles the inclusive $\mathrm{W}^{ \pm} \mathrm{W}^{ \pm} \mathrm{jj}$ EW production from the SM backgrounds. The signal region (SR) is divided into five bins of the signal BDT and five bins of the inclusive BDT. The nonprompt, $\mathrm{WZjj}$, tZq, and ZZ backgrounds have specific control regions (CR), which are used to constrain their normalizations in the ML fit; these CRs are divide into four $\mathrm{m}_{j j}$ bins.

The analysis sets an observed (expected) $95 \%$ confidence level upper limit on $\mathrm{W}_{\mathrm{L}}^{ \pm} \mathrm{W}_{\mathrm{L}}^{ \pm}$process of $1.17(0.88) \mathrm{fb}$, with the helicity eigenstates defined in the $\mathrm{W}^{ \pm} \mathrm{W}^{ \pm}$center-of-mass reference frame. The EW production of $\mathrm{W}^{ \pm} \mathrm{W}^{ \pm} \mathrm{jj}$ where at least one of the $\mathrm{W}$ bosons is longitudinally polarized reaches an observed (expected) significance of 2.3 (3.1) standard deviations (s.d.). Cross section's 

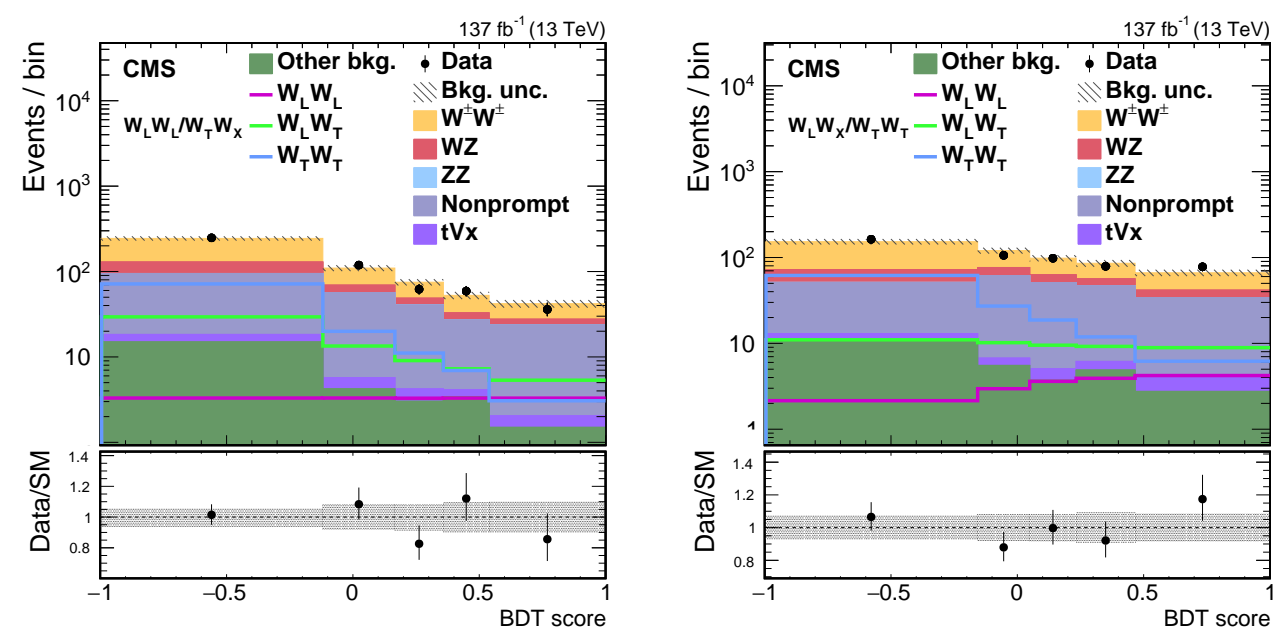

Figure 1: Post fit distributions of the output score of the signal BDT used for the $\mathrm{W}_{\mathrm{L}}^{ \pm} \mathrm{W}_{\mathrm{L}}^{ \pm} / \mathrm{W}_{\mathrm{X}}^{ \pm} \mathrm{W}_{\mathrm{T}}^{ \pm}$(left) and the $\mathrm{W}_{\mathrm{L}}^{ \pm} \mathrm{W}_{\mathrm{X}}^{ \pm} / \mathrm{W}_{\mathrm{T}}^{ \pm} \mathrm{W}_{\mathrm{T}}^{ \pm}$(right) cross section measurements. In the lower panel of each figure, the ratio of the number of events observed in data to that of the total SM prediction is shown, with the gray bands representing the uncertainties in the predicted yields and the vertical bars the statistical uncertainties in the data. From Ref.[2]

\begin{tabular}{ccc}
\hline Process & $\sigma \mathcal{B}(\mathrm{fb})$ & Theoretical prediction $(\mathrm{fb})$ \\
\hline $\mathrm{W}_{\mathrm{L}}^{ \pm} \mathrm{W}_{\mathrm{L}}^{ \pm}$ & $0.32_{-0.40}^{+0.42}$ & $0.44 \pm 0.05$ \\
$\mathrm{~W}_{\mathrm{X}}^{ \pm} \mathrm{W}_{\mathrm{T}}^{ \pm}$ & $3.06_{-0.48}^{+0.51}$ & $3.13 \pm 0.35$ \\
$\mathrm{~W}_{\mathrm{L}}^{ \pm} \mathrm{W}_{\mathrm{X}}^{ \pm}$ & $1.20_{-0.56}^{+0.53}$ & $1.63 \pm 0.18$ \\
$\mathrm{~W}_{\mathrm{T}}^{ \pm} \mathrm{W}_{\mathrm{T}}^{ \pm}$ & $2.11_{-0.47}^{+0.49}$ & $1.94 \pm 0.21$ \\
\hline
\end{tabular}

Table 1: Measured fiducial cross sections for the $\mathrm{W}_{\mathrm{L}}^{ \pm} \mathrm{W}_{\mathrm{L}}^{ \pm}$and $\mathrm{W}_{\mathrm{X}}^{ \pm} \mathrm{W}_{\mathrm{T}}^{ \pm}$processes, and $\mathrm{W}_{\mathrm{L}}^{ \pm} \mathrm{W}_{\mathrm{X}}^{ \pm}$and $\mathrm{W}_{\mathrm{T}}^{ \pm} \mathrm{W}_{\mathrm{T}}^{ \pm}$ processes with the helicity eigenstates defined in $\mathrm{W}^{ \pm} \mathrm{W}^{ \pm}$the center-of-mass reference frame. The uncertainty shown is the combination of the statistical and systematic uncertainties. The theoretical predictions include the $\mathrm{O}\left(\alpha^{6} \alpha_{S}\right)$ and $\mathrm{O}\left(\alpha^{7}\right)$ corrections to the $\mathrm{LO}$ cross sections; their uncertainties include statistical, PDF, and LO scale uncertainties. From Ref.[2].

measurements in the fiducial region are reported in Tab. 1 for the $\mathrm{W}^{ \pm} \mathrm{W}^{ \pm}$center-of-mass reference frame. Results are in agreement with SM predictions. Results are also obtained for the $\mathrm{W}^{ \pm} \mathrm{W}^{ \pm}$ helicity eigenstates defined in the initial-state parton-parton reference frame. More details on the analysis and the complete set of results are reported in Ref.[2].

\subsection{The cleanest channel: Most stringent aQGC limits}

The VBS production of a pair of Z bosons in association with two jets ( $\mathrm{ZZjj}$ ) has the lowest cross section among the VBS processes. The CMS collaboration claimed an evidence for this process using the full Run II data sample [3]. The analysis focuses on the fully leptonic final state, with four leptons and two jets. This signature reduces to negligible the experimental background. Indeed, background contamination in this channel arises mainly from QCD-induced ZZjj production and the loop-induced production of two Z bosons from a gluon-gluon initial state (ggZZjj). To separate the EW signal from the QCD-induced background, a matrix element discriminant $\left(\mathrm{K}_{\mathrm{D}}\right)$ has been built. 

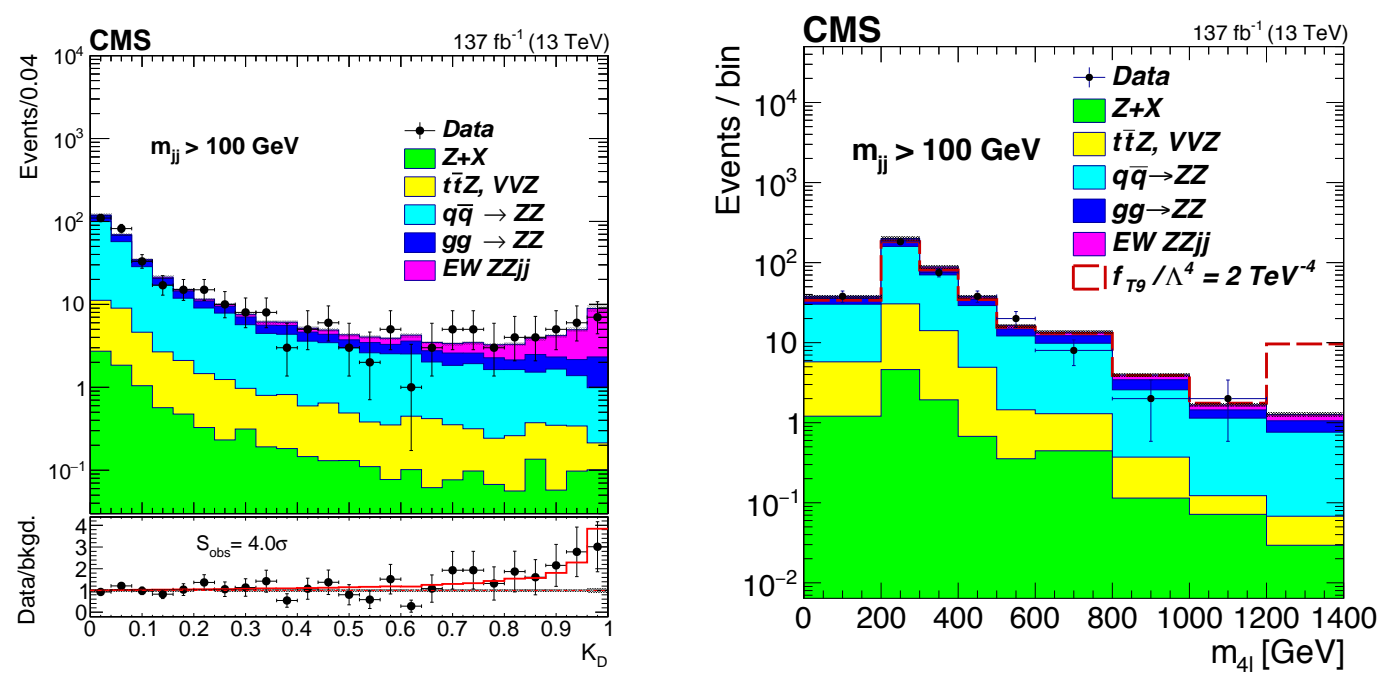

Figure 2: Postfit distributions for the the $\mathrm{K}_{\mathrm{D}}$ (left) and the four-lepton invariant mass for $f_{T 9} / \Lambda^{4}$ (right). Points represent the data, filled histograms the fitted signal and background contributions, and the gray band the uncertainties. The left lower panel shows the ratio of the number of events in the data to the total number of background events; the red line indicates the ratio of the fitted total distribution to its background-only component. From Ref. 2.

The $\mathrm{K}_{\mathrm{D}}$ discriminant distribution for events in the ZZjj SR, reported in the left panel of Figure 2, is used to extract the EW signal strength via an ML fit. The EW signal has an observed statistical significance of 4 s.d.; its measured fiducial cross section is $\sigma_{\text {fid }}=0.33_{-0.10}^{+0.11}$ (stat) ${ }_{-0.03}^{+0.04}$ (syst) fb. The signal strength of the EW+QCD ZZjj production is determined as well from a fit on the event counts. Cross-sections measurements are then extracted for both EW and EW+QCD processes. The results are in good agreement with SM expectations.

The invariant mass of the four leptons, shown in the right panel of Figure 2, is used to constraint the aQGCs parameters (Wilson coefficients) of the dimension 8 EFT operators T0, T1, $\mathrm{T} 2$, T8 and T9. The limit set at $95 \%$ confidence level on the neutral current operator T8 of $-0.43<f_{T 8} / \Lambda^{4}<0.43$ is the most stringent to date.

\subsection{The channel: First observation}

The first observation of the $\mathrm{Z} \gamma \mathrm{jj}$ EW production in the fully leptonic final state has been reported by the CMS collaboration using the full Run II data-set [4]. The final state is characterized by two leptons (with same sign charge and same flavor) from the $\mathrm{Z}$ boson decay, one photon $(\gamma)$, and two jets showing the typical VBS signature. The events are categorized into a signal region, enriched in signal events, and a CR, enriched in QCD-induced Z $\gamma \mathrm{jj}$ events, used to measure the normalization of this major background during the ML fit. SR and CR are split into the $e e / \mu \mu$ categories, and also with respect to the $\gamma$ being in the ECAL endcap or the ECAL barrel.

The ML fit is performed on SR and CR simultaneously; the SR is divided into $m_{j j}-\left|\Delta \eta_{j j}\right|$ bins (as in Fig. 3 left) and the CR into $m_{j j}$ bins. The Z $\gamma \mathrm{jj}$ EW production is observed with a signal significance well above the 5 s.d. Both the $\mathrm{EW}$ and $\mathrm{EW}+\mathrm{QCD}$ cross sections have been measured. 

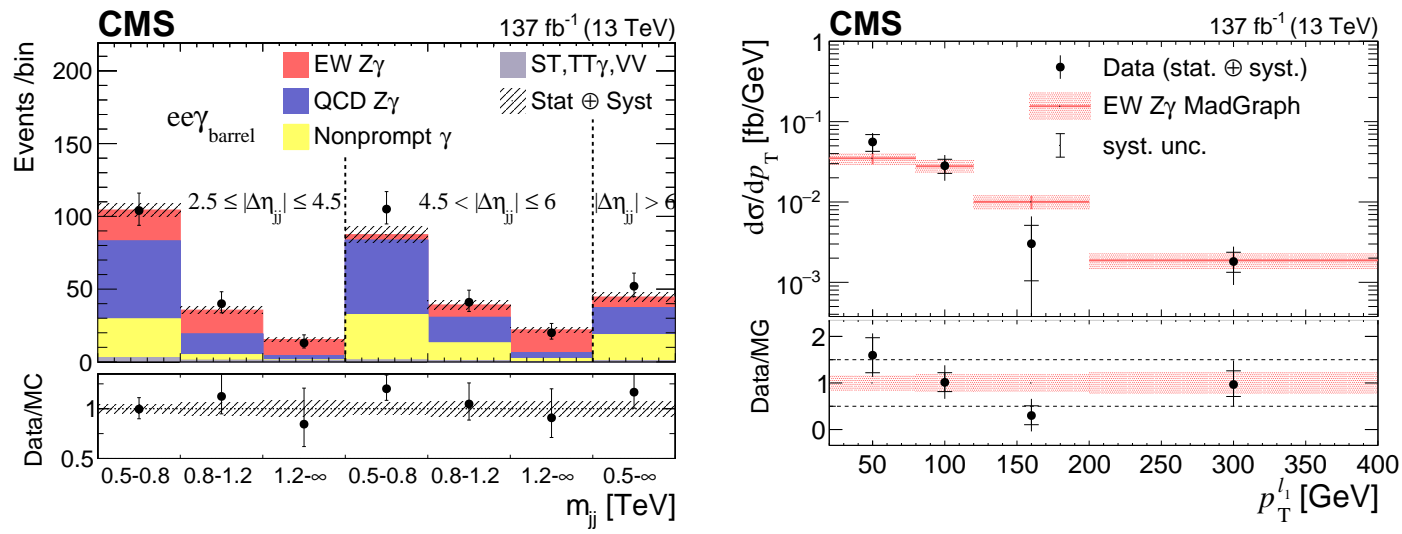

Figure 3: In the left panel:the post-fit distributions of the dielectron categories (with $\gamma$ in the barrel) in bins of $m_{j j^{-}}\left|\Delta \eta_{j j}\right|$. The black points represent the data, the error bars their statistical uncertainties. The hatched bands show the total uncertainties of the predictions. In the right panel: unfolded differential cross section as a function of the leading lepton $p_{\mathrm{T}}$; black points with error bars show the data and their statistical uncertainties, while the red bands represent the total theoretical uncertainties. From Ref.[4].

Differential cross sections measurements are performed as well, in bins of transverse momentum $p_{\mathrm{T}}$ of the leading lepton (Fig. 3), $\gamma$, and jets. Values measured agree with SM predictions.

Finally, constraints are set on the effective field theory dimension-8 operators M0 to M5, M7, T0 to T2, and T5 to T9, giving rise to aQGCs. The analysis provides the most stringent limit to date on the Wilson coefficient of T9, which is reported to be $-0.91<f_{T 9} / \Lambda^{4}<0.91$.

\section{Conclusion}

The vector boson scattering processes allow us to investigate the EW sector of the SM. Moreover, they are sensitive to possible anomalies in triple and quartic gauge couplings, which would be hints of new physics. VBS are rare processes, thus results obtained with the Run II data sample are still statistically limited. An improvement in the constraint of these processes is expected with the LHC Run III, starting in 2022, thanks to the larger data sample that will be collected.

\section{References}

[1] CMS Collaboration, The CMS experiment at the CERN LHC, JINST 3 (2008) S08004.

[2] CMS Collaboration, Measurements of production cross sections of polarized same-sign W boson pairs in association with two jets in proton-proton collisions at $\sqrt{s}=13 \mathrm{TeV}$, Phys. Lett. B 812 (2021) 136018, [hep-ex/2009. 09429].

[3] CMS Collaboration, Evidence for electroweak production of four charged leptons and two jets in proton-proton collisions at $\sqrt{s}=13 \mathrm{TeV}$, Phys. Lett. B 812 (2021) 135992 [hep-ex/2008.07013]. 
[4] CMS Collaboration, Measurement of the electroweak production of $Z \gamma$ and two jets in protonproton collisions at $\sqrt{s}=13 \mathrm{TeV}$ and constraints on anomalous quartic gauge couplings, Phys. Rev. D 104 (2021) 072001 [hep-ex/2106.11082]. 